\title{
Effect of Extent of Hippocampal and Amygdala Resection on Seizure Outcome in Patients with Refractory Epilepsy Secondary to Medial Temporal Sclerosis
}

\author{
${ }^{1}$ Sachin Bindal, ${ }^{2}$ Navneet Singla, ${ }^{3}$ Parampreet Singh, ${ }^{4}$ Manish Modi, ${ }^{5}$ Kokkula Praneeth, ${ }^{6}$ Manju Mohanty, ${ }^{7}$ Sameer \\ Vyas, ${ }^{8}$ Sunil K Gupta
}

\begin{abstract}
Introduction: Anterior temporal lobectomy with amygdalohippocampectomy is the most common surgical procedure for refractory epilepsy secondary to mesial temporal sclerosis. There is no consensus on whether the degree of hippocampal or amygdala resection has any influence on determining outcomes after epilepsy surgery. In this study, we assessed the seizure control and neuropsychological outcome and correlated these with the degree of surgical resection as determined on postoperative magnetic resonance imaging (MRI) in patients who had undergone surgery for refractory temporal lobe epilepsy.

Materials and methods: A total of 20 patients of refractory medial temporal lobe epilepsy were taken who underwent anterior temporal lobectomy with hippocampal and amygdala resection. Pre and Post-op clinical and neuropsychological assessment was done. Pre- and post-operative MR scans were compared for degree of hippocampal and amygdala resection. Seizure control was assessed based upon the degree of resection.
\end{abstract}

Results: Out of 18 patients with complete resection of the hippocampal body, 17 (94.4\%) patients had Engels class I outcome and one (5.6\%) patient had Engels class II outcome. But in patients with partial body resection, both the patients (100\%) had Engel's class II outcome. On complete resection of the tail, 12 patients had class I and one patient had class II outcome. When the tail of hippocampus was partially resected, or unresected resection five patients had class I but two patients had class II outcome.

Conclusion: It is required to achieve complete removal of pes hippocampus and hippocampal body for better seizure control and improvement in neuropsychological performance. The degree of the hippocampal tail or amygdala resection does not affect the outcome.

${ }^{1}$ Consultant, ${ }^{2}$ Assistant Professor, ${ }^{3,4,8}$ Professor, ${ }^{5}$ Post Doctoral Fellow, ${ }^{6}$ Additional Professor, ${ }^{7}$ Associate Professor

${ }^{1}$ Department of Neurosurgery, Shalby Hospital, Chandigarh

2,5,6,8 Department of Neurosurgery, Post Graduate Institute of Medical Education and Research, Chandigarh

${ }^{3,4}$ Department of Neurology, Post Graduate Institute of Medical Education and Research, Chandigarh

${ }^{7}$ Department of Radiodiagnosis, Post Graduate Institute of Medical Education and Research, Chandigarh

Corresponding Author: Navneet Singla, Department of Neurosurgery, Post Graduate Institute of Medical Education and Research, Chandigarh, India, Phone +917087008769, e-mail: drnavi2007@yahoo.co.in
Keywords: Hippocampal resection, Medial temporal sclerosis, Refractory epilepsy.

Abbreviations: MR-Magnetic resonance; MRI-Magnetic resonance imaging; AED-Anti-epileptic drug; MTLE-Medial temporal lobe epilepsy; MTLS-Medial temporal lobe sclerosis, EEG-Electroencephalogram; PGIMER-Post Graduate Institute of Medical Education and Research; DNET-Dysembryoplasticneuroepithelialtumour; IQ-Intelligence quotient; AHC-Amygdalohippocampectomy

How to cite this article: Bindal S, Singla N, Singh P, Modi M, Praneeth K, Mohanty M, Vyas S, Gupta SK. Effect of Extent of Hippocampal and Amygdala Resection on Seizure Outcome in Patients with Refractory Epilepsy Secondary to Medial Temporal Sclerosis. J Postgrad Med Edu Res 2019;53(1):11-16.

Source of Support: Nil

Conflict of Interest: None

\section{INTRODUCTION}

The World Health Organization (WHO) estimated that around 50 million people worldwide have epilepsy. ${ }^{1}$ Twothird of patients with epilepsy are adequately controlled with anti-epileptic medication with minimal or acceptable side-effects. ${ }^{2}$ Patients are considered to intractable or refractory epilepsy if at least two antiepileptic drugs (AEDs) have failed to control seizures despite being on maximally tolerated dose. ${ }^{3,4}$ Temporal lobe epilepsy constitutes almost two-thirds of all patients underwent surgery for drug-resistant epilepsy. ${ }^{5}$ Seizures often arise from mesial temporal structures including the amygdala, hippocampus, and parahippocampal gyrus. The term "mesial temporal sclerosis" describes pathological changes affecting the hippocampus, the amygdala, and the surrounding cortical areas. ${ }^{6}$ Hippocampal atrophy on MRI has been shown to correlate with the presence of hippocampal sclerosis. ${ }^{6,7}$

Surgery should be considered for seizure control in patients of medial temporal lobe epilepsy (MTLE) with uncontrolled seizures. ${ }^{8}$ Anterior temporal lobectomy with amygdalohippocampectomy is the most common surgical procedure for refractory epilepsy secondary to mesial temporal sclerosis. ${ }^{9}$ There is no consensus on whether the degree of hippocampal or amygdala resection has any influence on determining outcomes after epilepsy 
surgery. ${ }^{7}$ Hippocampal atrophy on MRI has been shown to correlate with intraoperative findings of hippocampal sclerosis. ${ }^{6}$

In this study, we assessed the seizure control and neuropsychological outcome and correlated these with the degree of surgical resection as determined on postoperative MR imaging in patients undergone surgery for refractory temporal lobe epilepsy.

\section{MATERIAL AND METHODS}

This study was a combined retrospective and prospective study conducted on patients of refractory temporal lobe epilepsy who underwent surgical treatment.

\section{Inclusion Criteria}

- Patients aged 12 years and above only were studied

- Patients labeled to have intractable epilepsy.

- Patients diagnosed to have medial temporal lobe sclerosis (MTLS).

- Patients with complete preoperative clinical, neuropsychological and radiological assessment including detailed clinical assessment of seizure severity, video EEG, memory and neuropsychological assessment and epilepsy protocol MRI.

- All patients who underwent surgery for MTLS at PGIMER in last 6 years.

- All patients who underwent only elective surgery were included.

\section{Exclusion Criteria}

- Patients having lesional epilepsy like temporal lobe tumors, DNET, vascular malformations, developmental defects, traumatic, ischemic, parasitic infections, etc were excluded.

- Patients without complete preoperative clinical, neuropsychological and radiological assessment were excluded

- Patients in whom adequate postoperative MRI were not available

- Patients not giving consent for inclusion.

- Patients with inadequate follow up were excluded. All patients were assessed by a team of neurologist, neurosurgeon, neuropsychologist, and neuro-radiologist.

\section{Clinical Assessment Included}

- History: Age of onset of a first seizure, description of seizure, the progression of seizure activity, response to antiepileptic drugs and course of changes in antiepileptic drugs including all increases in dosages and change of drug or addition of drugs.

- Physical examination: General physical examination, neurological examination, and other system examination performed.
- Standard EEG was done in all patients and video EEG was used to record the EEG continuously for 6 to 7 days. Video EEG was used in recording pre-ictal, ictal and post-ictal changes, which were studied by experienced interpreters.

\section{Neuropsychological Assessment}

Memory assessment (b) intelligent quotient (IQ) assessment. All patients were assessed for memory on the PGIMER memory scale which is validated for the Hindi speaking population. The IQ was assessed on Bhatia scale which consisted of two subsets Koh's block design test and Alexander Passalong test. This was done preoperatively as well as postoperatively.

\section{Neuroradiological Assessment}

Magnetic resonance imaging (MRI) was performed preoperatively as well as in the postoperative period. In the preoperative period, it was used in localization of the anatomical epileptogenic lesion. This included basic imaging: T1W and T2W images. Fat-sat and non-Fat-sat, 3D inversion recovery, magnetization-prepared rapid gradient echo (MPRAGE) imaging (Fig. 1).

Advanced imaging like MR volumetry, T2 relaxometry, inversion recovery, and MR spectroscopy.

The diagnosis of MTLS was made based on several of findings which included increased hippocampal signal intensity, hippocampal atrophy, atrophy of hippocampal collateral white matter, dilated ipsilateral temporal horn, reduced gray-white matter differentiation and associated temporal lobe atrophy.

\section{Operative Procedure}

Once the clinical history, video EEG and MRI changes corroborated and confirmed the localization of epileptogenic focus, the Patients were taken up for anteromedial temporal lobe resection + amygdalohippocampectomy (AHC). A standard temporal craniotomy under general anesthesia was done with bone exposed flush with the middle cranial fossa floor. Anterior temporal lobectomy was done with sparing of superior temporal gyrus. The length of temporal lobe resection was $4-4.5 \mathrm{~cm}$ on the dominant side, and $4.5-5.5 \mathrm{~cm}$ on a non-dominant side. The resection was always anterior to the vein of Labbe. After anterior temporal lobectomy, the temporal horn was opened, and amygdalectomy was done. The choroidal fissure was opened and hippocampectomy was done.

\section{Follow-up Period}

The follow-up period varied from a minimum of twelve months to 56 months with a mean of 24.2 months. 

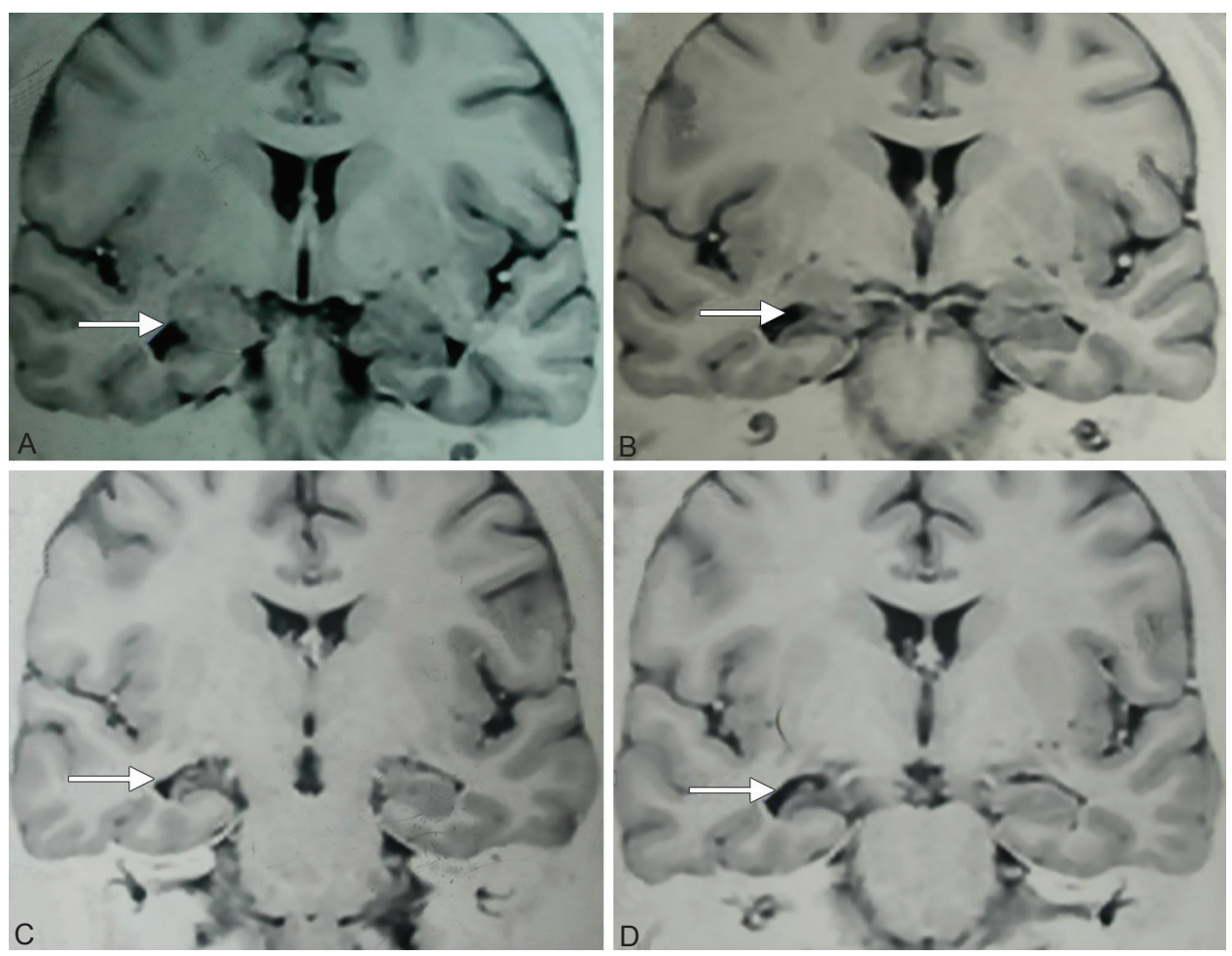

Figs 1A to D: Preoperative Inversion recovery sequence coronal MR images from anterior to posterior, showing Right MTLS, Loss of hippocampal volume, increased signal intensity, prominent temporal horn, decreased temporal lobe size. (A) amygdala; (B) pes hippocampus; (C) hippocampal body; (D) hippocampal tail

\section{Assessment at Follow-up}

- Assessment of seizure control:In the postoperative period, a detailed course of seizure control was recorded including all changes in semiology of seizures, changes in antiepileptic drugs prescribed and their dosages. All patients were assessed at 2 weeks, 1 month, 3 months, 6 months and one-year interval. The status at the last followup was used for statistical analysis. All retrospective patients were assessed during their regular follow up visits and from the record available with them. Seizure control/ outcome was assessed by Engel's classification. ${ }^{9}$

- Class 1-Free of disabling seizures

- Class 2-Rare disabling seizures (initially free of disabling seizures, but rare seizures now; rare disabling seizures since surgery; more than rare disabling seizures, but rare seizures for at least 2 years; nocturnal seizures only)

- Class 3-Worthwhile improvement (worthwhile seizure reduction; prolonged seizure-free intervals amounting to more than half the follow-up period, but not less than 2 years)

- Class 4-No worthwhile improvement (significant seizure reduction; no appreciable change; seizures worse)

\section{Neuropsychological Assessment}

The postoperative IQ and memory scores were compared with preoperative scores and the outcome was recorded as same, improved or deterioration in their memory and IQ scores.

\section{Neuroradiological Assessment}

Postoperative MRI (Fig. 2) was done in all patients. Post-operative image sequences included T1W, T2W, three-dimensional (3D) inversion recovery (IR) and T2 flair images. The neuroradiologist assessed each MR imaging sequences and determined the degree of surgical resection of pes hippocampus (complete/partial/ non-resected), hippocampal body (complete/partial/ non-resected) and tail(complete/partial/non-resected) and amygdala (complete/partial/non-resected). This was correlated with the seizure control and the neuropsychological outcome.

\section{RESULTS}

A total of 20 cases, who met the inclusion criteria were included. Age of the patients ranged from 16-44 years with a mean of 27.60 years. There were 11 male patients and 9 females. The age of onset of seizures was before 10 years of age in 8 patients, between 10-20 years of age in 9 patients and after the age of 20 years in 3 patients. Six patients presented with a history of febrile seizures in childhood and one patient had a history of Tubercular meningitis in childhood. Most of the patients presented with complex partial seizures. The complex partial 

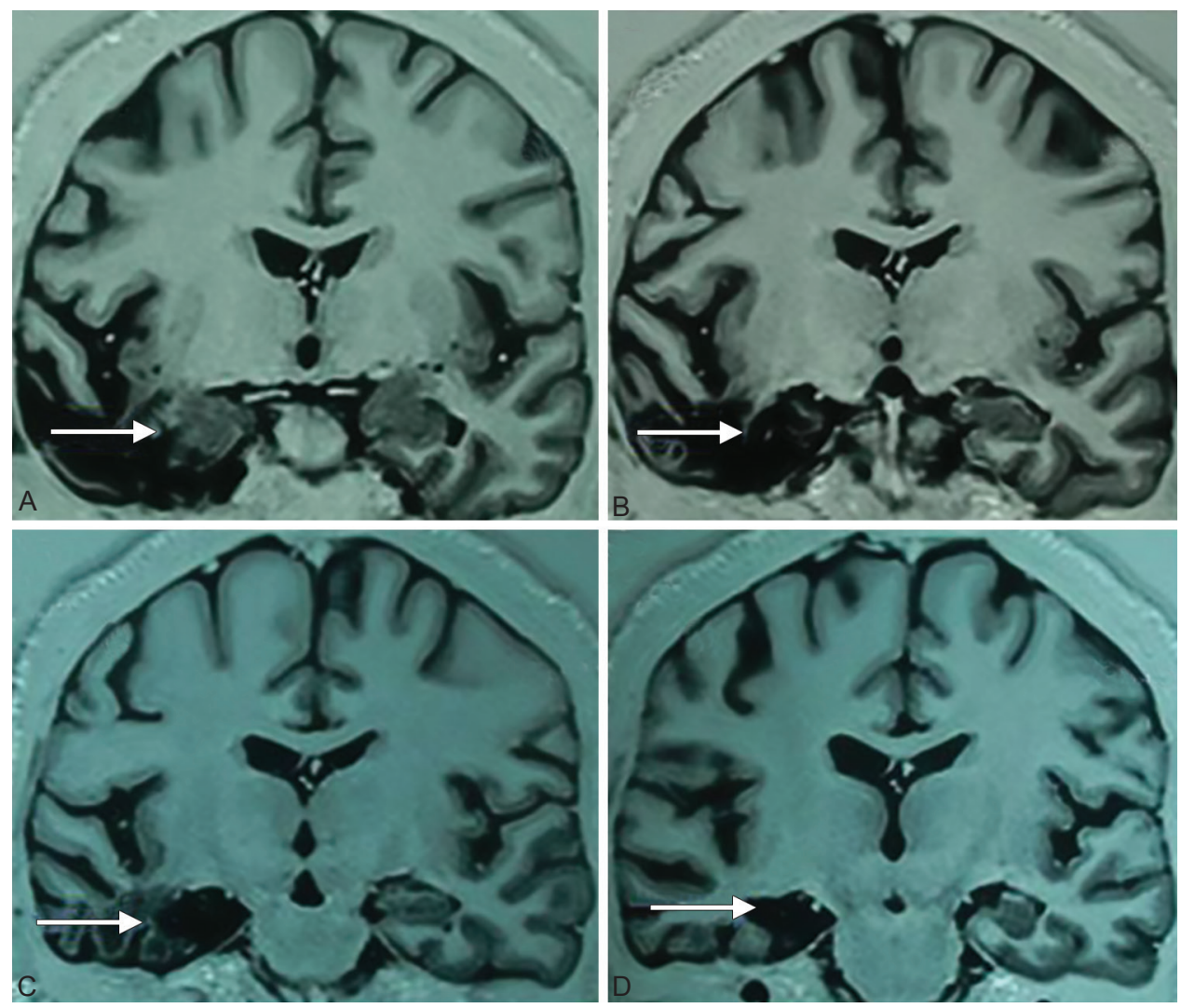

Figs 2A to D: Inversion recovery coronal MR images from anterior to posterior, showing postoperative changes of Right MTLR (A) partial amygdalectomy; (B) complete resection of pes hippocampus; (C) complete resection of hippocampal body; (D) complete resection of the hippocampal tail

seizures were alone $(n=4)$, with secondary generalization $(\mathrm{n}=6)$ or with associated generalized tonic-clonic seizures $(n=10)$. Patients taken up for surgery had seizure frequency varying from $2-3$ seizures/month to $>6$ seizures/day even on full doses of multiple AEDs. Eleven patients were diagnosed to have right MTLS while 9 had a left MTLS.

Two (10\%) patients had perioperative complications, one had a generalized tonic-clonic seizure on postoperative day 6, 7 and 11 due to operative-bed hematoma and one patient had post-operative delirium. Otherwise grossly there was good perioperative seizure control. The follow-up period varied from a minimum of twelve months to 56 months with a mean of 24.2 months.

Seizure outcome: Postoperatively till the last follow-up, 12 patients $(60 \%)$ had no seizure, and $8(40 \%)$ had seizures. Out of these 8 patients, who had seizures in follow up period, only 2 had seizures on same doses of AEDs without missing any dose. Rest all patients had seizures only on missing dose of AED or in the tapering of AEDs. Even in those getting seizures, the frequency of seizures was very less, and those too were non-disabling. Seventeen (85\%) patients had Engel's class I outcome with no seizures or occasional non-disabling seizures only on attempted drugs tapering or when they missed a dose or two. Two patients (10\%) had 2-3 seizures in follow-up on same doses of AEDs taken regularly had Engel's class II outcome. The third patient had seizures due to poor compliance; she often missed doses and follow-up.

- Neuropsychological outcome: 15 (75\%) patients had improvement in neuropsychological assessment. Five $(25 \%)$ patients remained the same. There was no worsening in neuropsychological status in any patient.

\section{Postoperative MRI Findings}

All patients had a complete resection of pes hippocampus. Complete hippocampal body resection was achieved in $18(90 \%)$ patients. In $2(10 \%)$ patients partial resection of the body of the hippocampus was achieved. Tail resection was complete in $13(65 \%)$ patients, partial in $4(20 \%)$ patients and remained unresected in $3(15 \%)$ of the patients. Amygdala resection was found to be complete in $3(15 \%)$ of the patients and was partial in rest $17(85 \%)$ patients.

\section{Seizure Outcome and Neuropsychological Outcome in Relation to Extent of Hippocampal and Amygdala Resection}

Out of 18 patients with complete resection of the hippocampal body, 17 (94.4\%) patients had Engels class I outcome, and one (5.6\%) patient had Engel's class II 
outcome. But in patients with partial body resection, both the patients (100\%) had Engel's class II outcome. On applying Fischer's exact test, the p-value was significant (0.016). Out of the 18 patients with complete body resection, on neuropsychological assessment, 15 (83.33\%) patients improved and $3(16.67 \%)$ remained same in neuropsychological assessment in postoperative assessment compared to preoperative assessment. But partial resection in 2 patients led to no improvement in neuropsychological parameters in both the patients.

On complete resection of the tail, 12 patients had class I and one patient had class II outcome. On partial resection, all four patients had class I outcome but when tail remained unresected one patient had to class I, but two patients had class II outcome. Though on applying tests of significance $p$-value was significant $(p=0.023)$ but this could be a mere coincidence as both the patients with unresected tails also had partially resected hippocampal body which was responsible for the poorer outcome. The postoperative neuropsychological assessment showed complete resection of tail leading of improvement in $11(84.62 \%)$ patients and no improvement in one $(15.38 \%)$ patient. Partial resection led to improvement in all 4 patients. Amount of amygdala resection was found to bear no significant relation with either Engel's outcome or outcome on neuropsychological assessment.

\section{DISCUSSION}

The present study was aimed at assessing the seizure outcome and neuropsychological status of patients undergoing surgery for MTLS and correlating it with postoperative MR evaluation and degree of resection.

\section{Seizure control}

In the present study, Engel's I outcome was achieved in 85\% of patients. This correlates well with the response achieved in literature. ${ }^{10,11}$ Tapering and reduction of antiepileptics was achieved in $80 \%$ of our patient. Patients who relapsed could be again made seizure free with restarting of anticonvulsants. Kim et al. also found that AED discontinuation could be achieved in $91 \%$ of patients with a successful outcome. ${ }^{12}$ Nearly $22 \%$ patients had a seizure relapse during reduction of anticonvulsant drugs and 7 (12\%) patients had a seizure after discontinuation of AEDs. But all patients became seizure free on the reinstitution of AEDs.

\section{Neuropsychological Outcome}

Sixteen (80\%) patients have shown improvement on neuropsychological assessment as compared to pre-op neuropsychological assessment, 4 (20\%) remained the same. However, Bandt et al. stated that postoperative neuropsychological assessment revealed a decline in verbal memory for the left AHC group while patients undergoing $\mathrm{AHC}$ on the right side showed improvement in memory. ${ }^{13}$ However, in our study, we found improvement in neuropsychological outcome in $80 \%$ of patients irrespective of the side of sclerosis. The improvement in neuropsychological status is probably linked to the absence/reduction of seizures in the postoperative period as well as to the reduction in antiepileptic drugs and drug dosage. It has been previously determined that the aggressive temporal lobe resection may lead to impairment of neuropsychological function and memory. ${ }^{7,11-13}$ We did not encounter any deficit in the neuropsychological testing or memory postoperatively. This is probably due to the result of careful patient selection and surgery.

\section{Correlation of Degree of Resection with Seizure Control and Neuropsychological Outcome}

There was a significant association between the degree of hippocampal body resection with seizure control and neuropsychological outcome. Patients with partial body resection had a poor seizure control as well as no improvement and in neuropsychological performance. Similarly, 2 out of 3 patients in whom tail remained unresected had Engel's class II outcome. But in both these patients body was also partially resected. The third one with an unresected tail who had complete body resection had Engel's class I outcome. Therefore unresected tail alone does not affect the outcome. The degree of resection (complete/partial) of amygdala also did not have any correlation with seizure control and neuropsychological outcome. Till date, there are no studies in the literature on the relation of the degree of resection with seizure control and neuropsychological outcome. There is only one study by Steven et al. where they found that the patients who had a complete resection of the hippocampus were more likely to achieve an Engel class I outcome. ${ }^{14}$ Our study has, therefore, demonstrated that resection of pes hippocampus, as well as the body of the hippocampus, is required for good seizure control. The degree of resection of the hippocampal tail or the amygdala possibly has no correlation with either the effectiveness of seizure control or with the neuropsychological status.

\section{Significance of Postoperative MRI}

By correlating the clinical outcome/seizure control and neuropsychological outcome with the degree of resection on postoperative MRI, we can obtain even better results and outcome by making necessary refinement in operative procedures. There is much more scope of further studies on using post-operative MRI in obtaining an even better outcome. There are no studies available correlating both the seizure control and neuropsychological outcome with the degree of resection on postoperative MRI. 
We conclude that it is required to achieve complete removal of pes hippocampus and hippocampal body for better seizure control and improvement in neuropsychological performance. The degree of the hippocampal tail or amygdala resection does not affect the outcome. But the numbers are small therefore a larger study is required to confirm the results.

\section{CONCLUSION}

We conclude that it is required to achieve complete removal of pes hippocampus and hippocampal body for better seizure control and improvement in neuropsychological performance. The degree of hippocampal tail or amygdala resection does not affect outcome. But the numbers are small therefore a larger study is required to confirm the results.

\section{REFERENCES}

1. Chang EF, Wang DD, Barkovich AJ, Tihan T, Auguste KI, Sullivan JE, et al. Predictors of seizure freedom after surgery for malformations of cortical development. Annals of neurology. 2011 Jul;70(1):151-162.

2. Wieser HG. Selective amygdalohippocampectomy: indications and follow-up. Canadian journal of neurological sciences. 1991 Nov;18(S4):617-627.

3. Berg AT. Defining intractable epilepsy. Advances in neurology. 2006;97:5-10

4. Berg AT, Vickrey BG, Testa FM, Levy SR, Shinnar S, DiMario $\mathrm{F}$, et al. How long does it take for epilepsy to become intractable? A prospective investigation. Annals of neurology. 2006 Jul;60(1):73-79.
5. Wiebe S. Epidemiology of temporal lobe epilepsy. Canadian Journal of Neurological Sciences. 2000 May;27(S1):S6-10.

6. Wieser HG. Presurgical Evaluation Protocols: University Hospital Zürich in Engel J Jr (ed): Surgical Treatment of the Epilepsies, ed 2. New York: Raven Press, 1993;738-738.

7. Commission on Classification and Terminology of the International League Against Epilepsy: "Proposal for revised clinical and electroencephalographic classification of epileptic seizures" Epilepsia 1981;22:489-501.

8. Engel J Jr, Van Ness PC, Rasmussen TB. Outcome with respect to epileptic seizures in Engel J, editor. Surgical treatment of the epilepsies. 2nd ed. New York: Raven Press; 1993;609-21.

9. Wieser HG. Behavioural consequences of temporal lobe resections in Trimble MR, Bolwig TG (eds): The Temporal Lobes and the Limbic System. Petersfield, UK: Wrightson Biomedical, 1992;169-188.

10. Wieser HG. Long-term seizure outcomes following Amygdalo-hippocampectomy. J Neurosurg. 2003;98:751-763.

11. Scheepers M, Kerr M. Epilepsy and behaviour. Current opinion in neurology. 2003 Apr 1;16(2):183-187.

12. Kim YD, Heo K, Park SC, Huh K, Chang JW, Choi JU, et al. Antiepileptic drug withdrawal after successful surgery for intractable temporal lobe epilepsy. Epilepsia. 2005 Feb;46(2):251-257.

13. Bandt SK, Werner N, Dines J, Rashid S, Eisenman LN, Hogan RE et al. Trans-middle temporal gyrus selective amygdalohippocampectomy for medically intractable mesial temporal lobe epilepsy in adults: seizure response rates, complications, and neuropsychological outcomes. Epilepsy Behav. 2013;28(1): 17-21.

14. Falowski SM, Wallace D, Kanner A, Smith M, Rossi M, Balabanov A, et al. Tailored temporal lobectomy for medically intractable epilepsy: evaluation of pathology and predictors of outcome. Neurosurgery. 2012 Jun 4;71(3):703-709. 